\title{
Strategies in cross-modality matching
}

\author{
ALLEN E. MILEWSKI and JAMES IACCINO \\ DePaul University, Chicago, Illinois 60614
}

\begin{abstract}
Subjects were tested on cross- and within-modality matching conditions with either a 5-sec or a 20-sec delay imposed between standard and comparison stimuli. Half the subjects were informed before the trial of what the comparison modality would be, while the others were not. There was a strong effect of instructions on the cross-modality conditions, but little on the within-modality conditions. The informed subjects showed better performance in the hapticvisual condition, while for the uninformed subjects, visual-haptic performance was better. The results suggest the importance of subjects' processing strategies in cross-modality performance.
\end{abstract}

The ability to integrate and compare information from different sensory modalities has most commonly been investigated with a cross-modality matching procedure. One finding of this procedure that has generated theoretical interest is that performance is often better for a cross-modality match in one direction than for one in the opposite direction. For example, Connolly and Jones (1970) found that matching a visual comparison stimulus to a previously presented kinesthetic standard (k-v) resulted in fewer errors than did matching a kinesthetic comparison to a visual standard ( $\mathrm{v}-\mathrm{k})$.

Asymmetries in cross-modality matching are of interest because, in addition to showing that intersensory transfer occurs, they can provide information about the processes involved. Connolly and Jones (1970) proposed an early model incorporating crossmodality asymmetries. They assumed: (1) the existence of modality-specific memory stores of which the visual store was superior in many situations (Posner, 1967); and (2) that internal transfer of information between modalities occurred prior to storage. While this model explains Connolly and Jones's findings and appears correct in emphasizing memory differences (Rose, Blank, \& Bridger, 1972; Garvill \& Molander, Note 1), its inadequacy as a general theory of intersensory integration has become clear. The predicted superiority of tasks in which vision is the comparison modality has been replicated (Cashdan, 1968; Freides, 1974; Jones \& Connolly, 1970; Marteniuk \& Rodney, 1979; Millar, 1972; Garvill \& Molander, Note 2). But there are numerous studies reporting no asymmetries (Diewaert \& Stelmach, 1977; Jones, 1973; Milner \& Bryant, 1970; Newell, Shapiro, \& Carlton, 1979; Rose et al., 1972) and asymmetries opposite to those predicted (Goodnow, 1971; Garvill \& Molander, Note 1). Attempts to explain this diversity of findings have concentrated almost entirely on stimulus differences. Explanations for the presence or direction of asymmetries have been based on whether or not the stimulus is spatial in nature (Pick, 1974), haptic or kinesthetic (Garvill \& Molander, Note 1), high in informational complexity (Freides, 1974, 1975) or presented in the context of a visible surround (Newell et al., 1979).

In their concentration on stimulus differences, all these explanations of cross-modality matching asymmetries have implicitly assumed transfer and storage to be mechanistic and automatic. However, since it is possible that subjects may have some control over these processes, other factors that may contribute to the asymmetry could be those related to subjects' processing strategies. One such variable may be the subjects' early awareness of what the comparison modality will be. For instance, results consistent with Connolly and Jones's model have often come from studies in which subjects have knowledge of the comparison modality through the use of blocked trials. It is quite possible that this knowledge permits or encourages transfer prior to storage, while the lack of knowledge may produce either variable or opposite results. Research in other contexts has shown the effect of expectations on memory performance (e.g., Tversky, 1969). In the cross-modality matching literature, only one investigation has directly manipulated knowledge of the comparison modality. Newell et al. (1979) compared matching performance under conditions of early and late instructions regarding the comparison modality. While these investigators argue that early knowledge of the comparison modality has no effect, their situation may not have provided an adequate test. In particular, they showed little or no evidence either for cross-modality asymmetry or for modality differences in within-modality comparisons (e.g., v-v and k-k). If it is assumed that the point at which subjects are given the task instructions determines which modality-specific store the information enters first, then null results are not surprising in situations in which the stores are equally retentive. In one experiment in which Newell et al. made visual information of the surround available, they did obtain cross-modality asymmetry. In that experiment, they also report an effect of instruction time, but fail to describe it fully. 
In order to understand the effects of subjects' processing strategies in cross-modality matching more fully, the present experiment was designed to compare performance under early and late instructions in a situation in which cross-modality asymmetries are obtained.

\section{METHOD}

Subjects

Forty male and female undergraduates participated in the study for experimental credit as part of the requirements of an introductory psychology course.

\section{Stimull and Apparatus}

Four line lengths were used as stimuli: $11.75,12.38,13.02$, and $13.65 \mathrm{~cm}$. Within-modality pilot matchings showed these distances to result in a medium range of errors, and they were used to avoid ceiling and floor effects. They were presented visually for about 5 sec as dark, single horizontal lines on white index cards. They were presented binocularly at eye level at a distance of approximately $60 \mathrm{~cm}$. The same set of lines was used as standard and comparison stimuli. Haptic presentation was accomplished by adjusting the length of a .6-cm groove in the middle of a flat board. The subject's index finger was always placed at the same starting position, and he or she was instructed to actively explore the line length for approximately $5 \mathrm{sec}$. The experiment was run in a welllit room. The stimulus conditions were chosen to maximize the possibility of obtaining asymmetries.

\section{Design and Procedure}

Following five practice trials, each subject received 64 matching trials. On each trial, the subject made a verbal same-different judgment without feedback. The stimulus line lengths for each trial were selected randomly. On either the first or the last 32 trials, there was a 5-sec delay between the removal of the standard and presentation of the comparison. The other 32 trials were run with a 20-sec delay. Each trial was separated by 3 to $5 \mathrm{sec}$. The order of delay conditions was counterbalanced across subjects. Within each delay, there were eight trials on each of four conditions $(v-v, h-h$, $v-h, h-v)$. They were ordered randomly, with the restriction that each condition have equal frequency. Half of the subjects were assigned to an informed group and were instructed at the beginning of each trial what the standard and comparison modalities would be. The other half of the subjects (the uninformed group) did not receive this information prior to stimulus presentation. In all other respects, the two groups were treated identically.

\section{RESULTS}

The mean number of correct matches out of eight in each condition are shown in Table 1 . These data were analyzed in a 2 (instructions) $\times 2$ (delay) $\times 4$ (condi-

Table 1

Mean Number of Correct Responses (of Eight) for the Four Conditions Across Delay and Information Group

\begin{tabular}{|c|c|c|c|c|c|c|}
\hline \multirow[b]{2}{*}{$\begin{array}{c}\text { Condi- } \\
\text { tion }\end{array}$} & \multicolumn{3}{|c|}{ Informed } & \multicolumn{3}{|c|}{ Uninformed } \\
\hline & $\begin{array}{l}5-\mathrm{Sec} \\
\text { Delay }\end{array}$ & $\begin{array}{c}20-S e c \\
\text { Delay } \\
\end{array}$ & Mean & $\begin{array}{l}5 \text {-Sec } \\
\text { Delay } \\
\end{array}$ & $\begin{array}{c}20-S e c \\
\text { Delay } \\
\end{array}$ & Mean \\
\hline $\mathbf{v - v}$ & 7.30 & 7.00 & 7.15 & 7.05 & 6.75 & 6.90 \\
\hline$v-h$ & 5.15 & 4.35 & 4.75 & 6.25 & 5.60 & 5.92 \\
\hline$h-v$ & 6.20 & 5.85 & 6.02 & 4.85 & 4.10 & 4.47 \\
\hline h-h & 5.45 & 4.55 & 5.00 & 5.85 & 5.20 & 5.52 \\
\hline
\end{tabular}

Table 2
Results of a Post Hoc Newman-Keuls Test on the Eight Combinations of Condition and Information Groups

\begin{tabular}{|c|c|c|c|c|c|c|c|c|}
\hline & U h-v & I v-h & I h-h & U h-h & $\mathbf{U} \mathbf{v}-\mathbf{h}$ & I h-v & $\mathrm{U} v-\mathrm{v}$ & I v-v \\
\hline U h-v & & & & & * & $*$ & * & * \\
\hline I $\mathrm{v}-\mathrm{h}$ & & & & & * & $*$ & * & * \\
\hline I $h-h$ & & & & & & & $*$ & $*$ \\
\hline U h-h & & & & & & & * & $*$ \\
\hline U v-h & & & & & & & & * \\
\hline I $h-v$ & & & & & & & & \\
\hline$U \mathrm{v}-\mathrm{v}$ & & & & & & & & \\
\hline I v-v & & & & & & & & \\
\hline
\end{tabular}

Note $-I=$ informed group; $U=$ uninformed group.

*Significant at $p<.05$ or better.

tions) analysis of variance. There was a significant effect of delay $[F(1,38)=14.16, p<.001]$, indicating better overall performance for the 5-sec delay compared with the 20 -sec delay. There was also a reliable main effect of conditions $[\mathrm{F}(3,114)=32.67, \mathrm{p}<.001]$ and a significant interaction between conditions and instructions $[F(3,114)=14.76, p<.001]$. A post hoc Newman-Keuls test compared the eight combinations of conditions and instructions for both delays combined. The results of this test are shown in Table 2. Also, separate three-factor analyses were performed for the within-modality conditions ( $v-v$ and $h-h)$ and for the cross-modality conditions ( $v-h$ and $h-v)$. For the within-modality analysis, there were significant main effects of delay $[F(1,38)=9.71, p<.01]$ and of conditions $[\mathrm{F}(1,38)=30.05, \mathrm{p}<.001]$, indicating deteriorating performance with delay and also that, regardless of instructions, the v-v condition was superior to the $h-h$ condition. For the cross-modality analysis, there was again a main effect of delay $[F(1,38)$ $=10.09, \mathrm{p}<.01]$. While there was no overall difference between conditions, the condition $x$ instruction interaction was highly significant $[F(1,38)=25.00$, $\mathrm{p}<.001]$. This interaction suggests better performance in the $h-v$ condition for the informed subjects, but the opposite for uninformed subjects.

\section{DISCUSSION}

The results of the present experiment are of interest in two respects. First, they replicate earlier studies in showing that cross-modality asymmetries can be obtained reliably, at least under some conditions (see Freides, 1974). Second, they demonstrate that subjects' early knowledge of the comparison modality can affect performance in specific ways. For example, knowledge of the comparison modality affected the cross-modality conditions, but had no effect on the within-modality conditions. Moreover, while it did not affect overall performance, this knowledge reversed the direction of the cross-modality asymmetry. Under informed conditions, h-v performance was better than $\mathrm{v}$-h performance. Under informed conditions, the opposite was true. 
To explain the present results, we posit, like Connolly and Jones (1970), a vision-specific memory store that is, under the present conditions, superior to its haptic counterpart. However, this explanation differs from earlier ones in assuming situation-specific strategies. Under informed conditions, when subjects know which modality to transfer information to, they do so, even when it means storing information in the inferior haptic memory. To describe performance under uninformed conditions, at least three explanations are possible. First, perhaps when subjects do not know what the comparison modality will be, they may retain information in the initial memory store until transfer is required for a match. $\mathrm{H}-\mathrm{v}$ performance would be inferior to $v$-h performance because of rapid haptic memory decay. An alternative is to assume that, when uninformed, subjects form dual memory codes, storing information both in the standard modality and the comparison modality. $\mathrm{H}-\mathrm{v}$ performance by this explanation would be inferior, not because of memory decay, but because haptic storage requires more processing capacity (Posner, 1967). In this case, there would be less capacity available for a complete $\mathrm{h}-\mathrm{v}$ transfer. Yet another possibility is that haptic storage is more variable or less precise than visual storage. These latter two explanations appear preferable because they are consistent with other aspects of the present results. First, there was no effect of memory delay on either the asymmetries or instruction effects. A model based on differential decay rates would have predicted a delay effect. Second, while not statistically significant, cross-modality performance in uninformed conditions appears to be consistently worse than in the corresponding within-modality conditions. Again, the first model, based only on memory decay, would have predicted equal performance in cross- and within-modality conditions.

These results and explanation of cross-modality asymmetries are not in conflict with previous models based on stimulus differences. It is likely that there are numerous factors affecting cross-modality transfer and storage. This demonstration of instruction effects suggests that, at least under some stimulus conditions, subjects' processing strategies may be one of these factors. It should be noted that the present results provide no direct evidence that subjects' processing strategies affect cross-modality matching. However, direct manipulation of subjective memory strategies may be difficult and may not result in performance differences with subjects beyond middle childhood (Flavell, 1970).

Many aspects of the present model are necessarily vague. It is not certain, for example, if the strategies hypothesized here are voluntary or automatic, how they interact with stimulus conditions, or, indeed, what kind of transfer takes place. However, the re- sults of the present study indicate that processing strategies should be taken into account in future models of cross-modality performance.

\section{REFERENCE NOTES}

1. Garvill, J., \& Molander, B. Effects of retention intervals, standard modality and comparison modality on matching of form (Umeå Psychological Reports, No. 57). Umeă, Sweden: University of Umeå, 1972.

2. Garvill, J., \& Molander, B. Asymmetric effects in cross-modal transfer (Umeă Psychological Reports, No. 3). Umeå, Sweden: University of Umeå, 1968.

\section{REFERENCES}

Cashdan, S. Visual and haptic form discrimination under conditions of successive stimulation. Journal of Experimental Psychology, 1968, 76, 215-218.

Connolly, K., \& Jones, B. A developmental study of afferentreafferent integration. British Journal of Psychology, 1970, 61, 259-266.

Diewaent, G. L., \& Stelmach, G. E. Intra-modal and intermodal transfer of movement information. Acta Psychologica, $1977,41,119-128$.

Flavell, J. Developmental studies of mediated memory. In H. Reese \& L. Lipsitt (Eds.), Advances in child development and behavior (Vol. 5). New York: Academic Press, 1970.

Freides, D. Human information processing and sensory modality: Cross-modal functions, information complexity, memory, and deficit. Psychological Bulletin, 1974, 81, 284-310.

Freides, D. Information complexity and cross-modal functions. British Journal of Psychology, 1975, 66, 283-287.

Goodnow, J. The role of modalities in perceptual and cognitive development. In J. Hill (Ed.), Minnesota Symposia on Child Psychology (Vol. 5). Minneapolis: University of Minnesota Press, 1971.

JoNES, B. When are vision and kinaesthesis comparable? British Journal of Psychology, 1973, 64, 587-591.

Jones, B., \& Connolty, K. Memory effects in cross-modal matching. British Journal of Psychology, 1970, 61, 267-270.

Marteniuk, R. G., \& Rodney, M. Modality and retention effects in intra- and cross-modal judgments of kinaesthetic and visual information. British Journal of Psychology, 1979, 70, 405-412.

Millar, S. The development of visual and kinaesthetic judgments of distance. British Journal of Psychology, 1979, 63, 271-282.

Milner, A. D., \& Bryant, P. E. Cross-modal matching by young children. Journal of Comparative and Physiological Psychology, $1970,71,453-458$.

Newell, K. M., Shapiro, D. C., \& Carlton, M. J. Coordinating visual and kinaesthetic memory codes. British Journal of Psychology, 1979, 70, 87.96.

Pick, H. L. Visual coding of nonvisual information. In R. B. MacLeod \& H. L. Pick (Eds.), Perception: Essays in honor of James J. Gibson. Ithaca, N.Y: Cornell University Press, 1974.

Posner, M. Characteristics of visual and kinesthetic memory codes. Journal of Experimental Psychology, 1967, 75, 103-107.

Rose, S., Blank, M. S., \& Bhidgen, W. Intermodal and intramodal retention of visual and tactual information in young children. Developmental Psychology, 1972, 6, 482-487.

TVersky, B. Pictorial and verbal encoding in a short-term memory task. Perception \& Psychophysics, 1969, 6, 225-233.

(Manuscript received June 8, 1981; revision accepted for publication December 2, 1981.) 